\title{
Evaluation of Learning Samples for Fish Recognition with Machine Learning
}

\author{
Kazutaka Sagara ${ }^{\text {a,* }}$, Kousuke Katada ${ }^{\text {a }}$, Lifeng Zhang ${ }^{\text {a }}$ \\ ${ }^{a}$ 1-1 sensui-cho Tobata-ku, Kitakyushu city, Fukuoka, Japan 804-8550 \\ Kyusyu Institute of Technology \\ *Corresponding Author: kattaka4645@gmail.com
}

\begin{abstract}
Recent years, the invasive exotic fishes are destroying the ecosystem of the native fishes has happened all over the world. There are many approach are considered to prevent this happening, but no effective measure is figured out to eradicate the exotic fish by now. Therefore we propose a new method by using image processing. This new method is aimed to distinguish the specified exotic fish from the local native fish automatically by using underwater camera. In this study, we perform fish recognition to discriminate whether fish exists or not in the image. This process is an important pretreatment for determining fish species automatically. Fish recognition is more difficult than human face recognition because fish has no symmetry and have not an obvious unique feature can be used. Especially the image taken in underwater could cause a large quality distortion. In order to evaluate the efficiency of exist algorithm in this situation, in this paper, we statistically examine that what kind of learning methods applied for what kind of learning samples made from fish body features can bring good results.
\end{abstract}

Keywords: Fish recognition, learning samples, Haar-Like feature, machine learning, AdaBoost.

\section{Introduction}

As described in the abstract, the invasive exotic fish cause a variety of problems all over the world [1]. For example, eating native fish, genetic pollution by mating and bringing parasite and illness which have never existed in the local region. These problem adversely affect the biodiversity, agriculture and forestry, fishery, and human healthy. Actually, many countries are affected by such problems. In particular black bass is the most famous creature among the exotic fish in Japan. Black bass is very berserk and eat a large amount of fish. Therefore the ecosystem of native fish is subjected to a large damage if someone brings the black bass to the river or lake illegally. This fish has given a variety of influence in Japan. Figure.1. shows the total catch of Lake Biwa. As you can see, the mount of fish catches is decreasing year by year. This is said cause by blacks bass. Now, fishing black bass by people hands or pulling out the water of the pond and lake is the countermeasure for exotic fish. However this method requires a lot of cost and personnel. It is difficult to say that efficient. Therefore, we need to suggest a more efficient method. The method is that we set up an underwater camera in the ponds and lakes, and use the proposed algorithm to discriminate whether exotic fish or native fish exist. If a fish passing through in the front of the camera is exotic fish, we can vanish it automatically by an underwater robot.

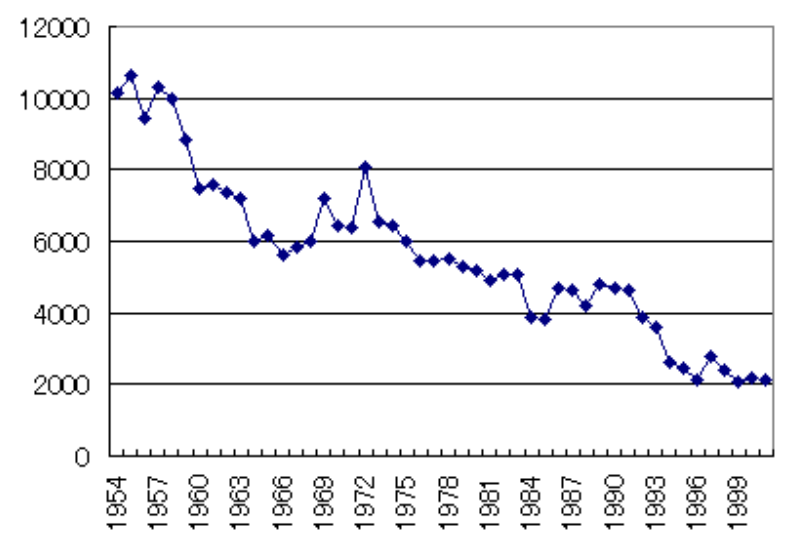

From:http://www.wwf.or.jp/activities/2009/09/679714.html(2015.7.30)

Fig.1. Total catch changing of Lake Biwa (ton)

Currently, object detection technology is making 
progress day by day. For example, human face detection is a very success one of them. Actually, the camera equipped with a face detection sensor has been sold all over the world. However recognition of a fish is more difficult than human face, because human face is characterized by symmetry and the geometric structure is not changed so much. Objects with non-symmetry are difficult to take the feature point. In this study, we improve the accuracy of the object detection with non-symmetry by attaching conditions. Through this study, we research the new method, which discriminate exotic fish.

\section{Method}

In this research, our destination is to select a proper area from fish body image as a learning pattern. To perform this evaluation, we use an existing machine learning algorithm called AdaBoost. It is described in following.

\subsection{Flow of object recognition}

Learning and recognition are two phases of object recognition. Flow of Adaboot shows in Fig.2.

In learning phase, in order to construct the data of learning result, collection of a large amount of positive images and negative images are needed. Then, feed these images into learning program to extract features. At here Haar-Like features are used. Next, learn by using these features with an iterative procedure which called “AdaBoost". Finally, gets the data of learning result.

For recognition phase, first, extract features from the input image. Second, match the input image and data with data of learning result. Finally, it outputs the result. In this study, it focuses on the learning phase and improve the accuracy. We explain below about these details.

\subsection{Learning image}

There are two types of learning images. One is positive image that the target object is included in it. The other is negative image that the target object must not be included in it. The negative images can be background images or any non-target objects' image.

\subsection{Haar-Like features}

Haar-Like features are scalar quantity which is obtained as a difference value of the average brightness in rectangular area. This value represents the intensity of the brightness gradient. It doesn't dependent on absolute brightness value and extract features which corresponds to the texture. Haar-Like features are shown Fig.3. The difference of the luminance values between white and black part define as features. We show the Eq. (1).

$$
\mathrm{H}\left(\mathrm{r}_{1}, \mathrm{r}_{2}\right)=S\left(\mathrm{r}_{1}\right)-S\left(\mathrm{r}_{2}\right)
$$

where, $S\left(\mathrm{r}_{1}\right)$ is average brightness of the white areas, $S\left(\mathrm{r}_{2}\right)$ : is the average brightness of the black areas.

Haar-Like features have three features pattern, "Edge features", "Line features", "Center-surround features". It is possible to extract the fine features by making full use of them. Haar-Like features pattern is shown figure.4.

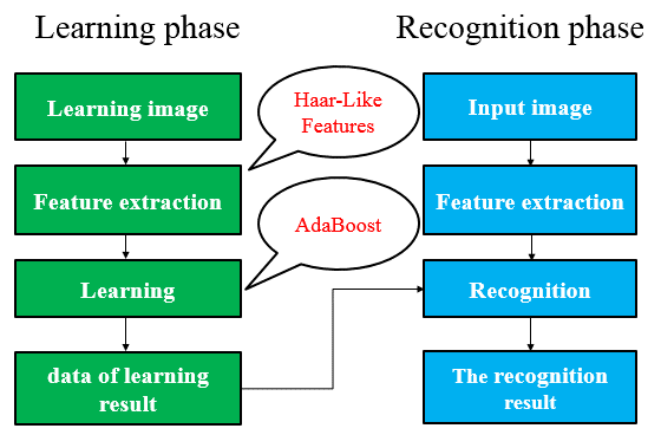

Fig.2. Flow of image recognition

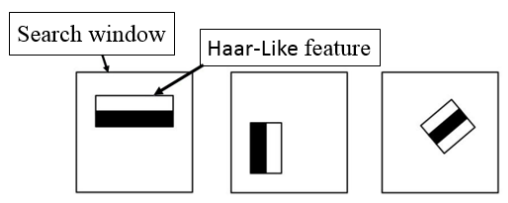

Fig.3. Haar-Like features

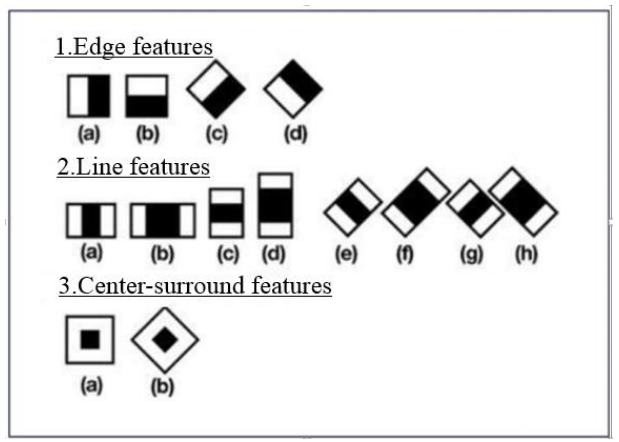

Fig.4. Haar-Like features pattern

\subsection{AdaBoost [5]}

To classify the fish from other object by using these Haar-Like features, it is necessary to select the correct 
Haar-Like feature from all of the candidates. AdaBoost is used here. It is algorithm that makes a strong classifier by combining the weak classifiers which has a low individual capacity. It will take a lot of time if applying all of the Haar-like patterns randomly to make weak classifiers. But using this algorithm, it is possible to determine the meaningful Haar-Like pattern candidates. Therefore, using these candidates to make weak classifiers will save a lot of time.

\section{(a) Pretreatment}

First prepare the $N$ number of learning samples. $\left(x_{1}, y_{1}\right) \ldots,\left(x_{N}, y_{N}\right)$. Then $x_{i}$ is images, and $y_{i}$ is class label. Class label is indicator of whether the image is correct or not. For example, when the detection of human, the human image is given label of +1 and no-human image is given label of -1 .

(b) Initialization of the weights of the learning sample

The initial weights of each sample $D_{t}(i)$ are set to equal. It is shown in the expression (2).

$$
D_{t}(i)=\frac{1}{N}
$$

\section{(c) Candidate of weak classifiers}

Candidate of weak classifiers are basically constructed randomly. However we must choose the classifiers which the error rate is 0.5 or less. In this study, we design weak classifiers based on Haar-Like features as shown in equation (3), because it uses Haar-Like features.

$$
h_{t}(x)=\left\{\begin{array}{lr}
1 & p \cdot v(x)>p \cdot \theta \\
-1 & \text { othewise }
\end{array}\right.
$$

$h_{t}: t$ round of weak classifiers

$\theta:$ Features

$v$ : Threshold

$p$ : Variable that sets the inequality of direction( 1 or -1$)$

(d) The calculation of the error rate

Calculate the error rate $e_{t, m}$ for a weak classifier.

If response value of the weak classifiers $h$ and class label is difference, add the weights of the samples. It is shown in the Eq. (4).

$$
e_{t, m}=\sum_{i: h_{t}\left(x_{i}\right) \neq y_{i}}^{N} D_{t}(i)
$$

Also if the error rate exceeds 0.5 , invert the parity $\mathrm{p}$ of weak classifiers. It is shown in the figure.5.

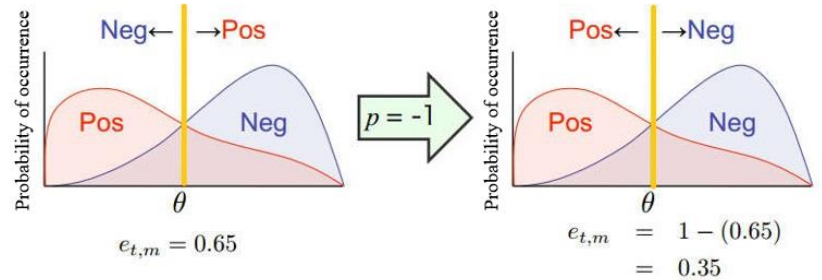

From:http://www.vision.cs.chubu.ac.jp/joint_hog/pdf/HOG

+Boosting_LN.pdf (2015.7.30)

Fig.5.Reversal of parity $\mathrm{p}$ of weak classifiers

(e) Calculate the weight $\propto_{t}$ for the adopted weak classifiers

It is shown in the Eq. (5) which calculates the weight $\propto_{t}$ from error rate.

$$
\propto_{t}=\frac{1}{2} \ln \left(\frac{1-e_{t}}{e_{t}}\right)
$$

(f) Update the weights of learning samples

Eq. (6) shows the update of weights of learning samples $D_{t}$.

$$
D_{t+1}(i)=D_{t}(\mathrm{i}) \exp \left(-\propto_{t} y_{t} h_{t}\left(x_{i}\right)\right)
$$

(g) Normalization of the weights of the learning sample Normalize the weights of learning samples $D_{t}$ is shown in Eq.(7)

$$
D_{t+1}(i)=\frac{D_{t+1}(i)}{\sum_{i=1}^{N} D_{t}(\mathrm{i}) \exp \left(-\propto_{t} y_{t} h_{t}\left(x_{i}\right)\right)}
$$

(h) Construction of the final classifier

Give the weights to all weak classifier and take a majority. For example, when determining the human, it judges human if $\mathrm{H}$ is higher than threshold $\lambda$. It is shown in the expression (8).

$$
\mathrm{H}(\mathrm{x})=\operatorname{sign}\left[\sum_{t=1}^{T} \propto_{t} h_{t}(x)-\lambda\right]
$$

$\lambda$ : Detector threshold

\subsection{Experiment}

We created several original object classifiers by using different learning pattern using the previously mentioned algorithm. Then using these classifiers to recognize the fish to see how change of the detection rate by the different learning pattern. The detector is set to surround the object with a red frame if the detecting result is positive. And if the surrounded location has a positive object in there, we treat it as a correct result, if not, a false recognition.

First we prepare positive images and negative images 1,000 pieces for each. Figure 6 and Fig .7 show the examples of the positive image and negative image respectively. 

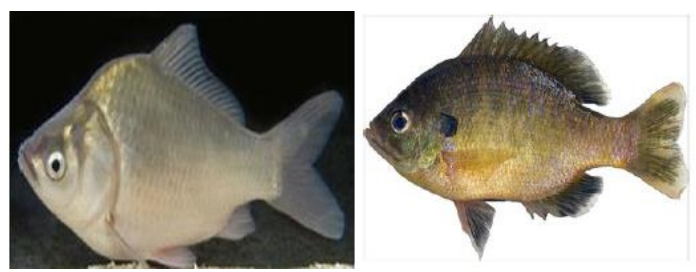

Fig.6: examples of the correct image

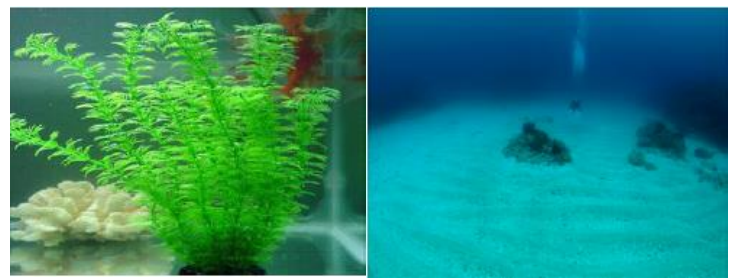

Fig.7: examples of the non-correct image

As mentioned above, to detect a deformed object is difficult because there are variations in feature. Therefore we select five different kinds of body feature as positive image to make the classifier.

(1) Entire of the body pattern.

(2) Face pattern.

(3) Eye pattern.

(4) Body surface pattern.

(5) Dorsal fin pattern.

Figure 8(1)-(5) show the examples of the learning part respectively.

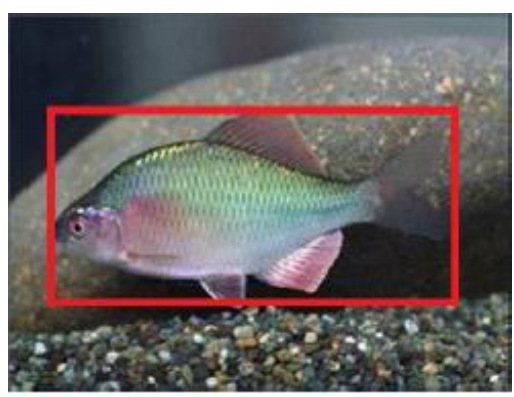

(1) Body pattern

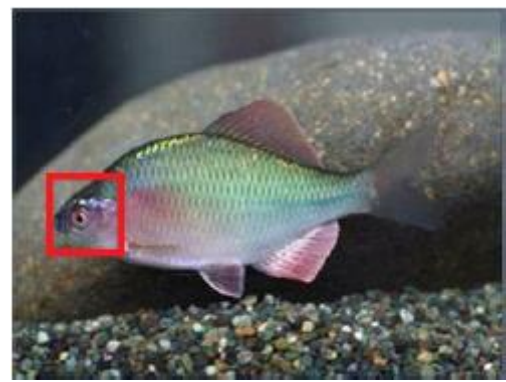

(2)Face pattern

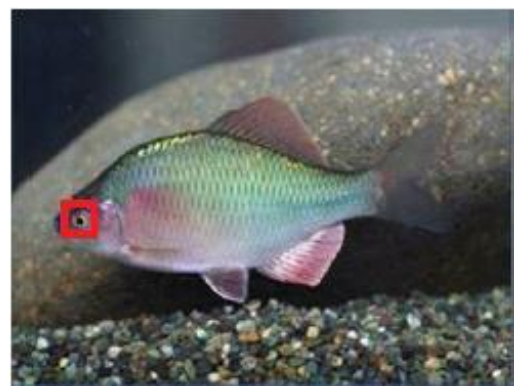

(3) Eye pattern

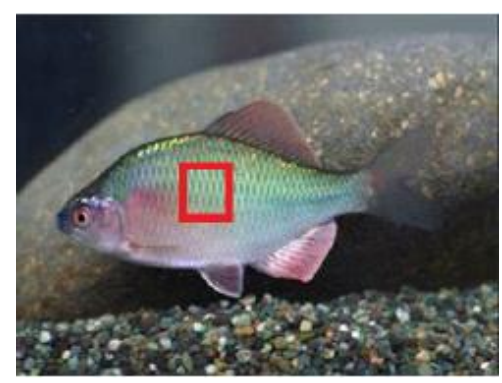

(4) Body surface pattern

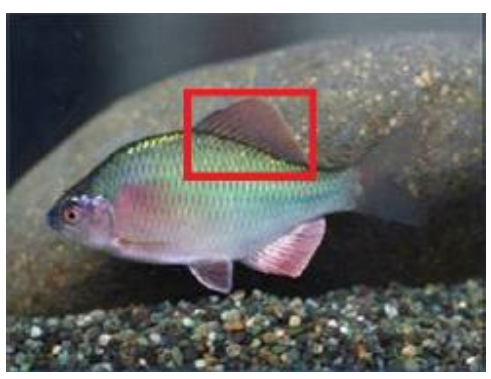

(5) Dorsal fin pattern

Fig.8: examples of the learning part

After learning, we verify one by one by using 100 pieces of fish images collected from the Internet.

Eq. (9) shows the definition of numerical accuracy.

$$
\begin{aligned}
& \text { Recognition rate }= \\
& \quad \frac{\text { Correct detection number }}{100}(\%) \\
& \text { False recognition rate }= \\
& \frac{\text { The number of detected false }}{100}(\%)
\end{aligned}
$$

\section{Result}

\subsection{Learning result of the entire body pattern}

In this experiment, 64 of 100 images are accurately recognized. Figure 9 shows the examples of the correct and the false recognition results. 


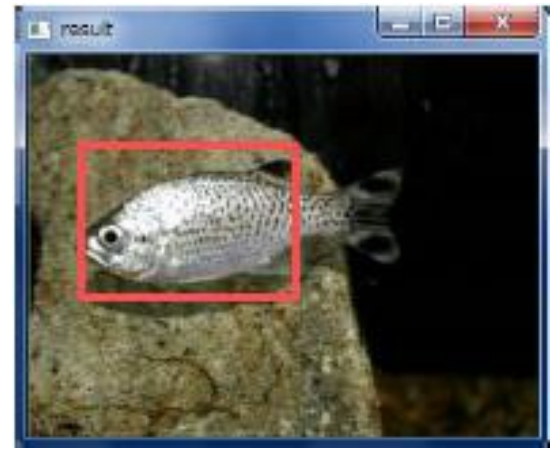

(1) Examples of recognition

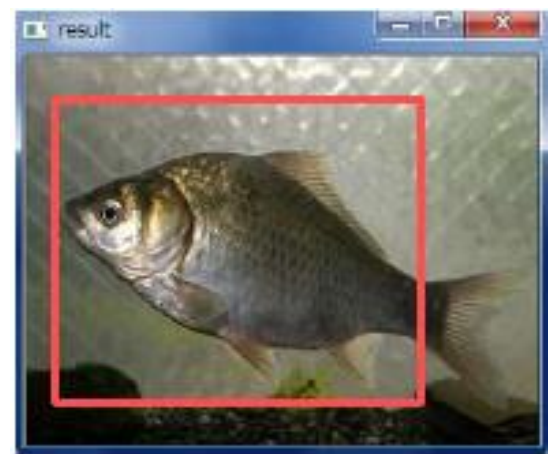

(2) Examples of recognition

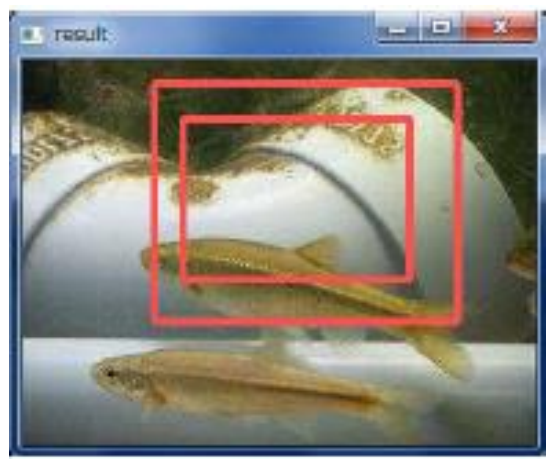

(3) Examples of false recognition

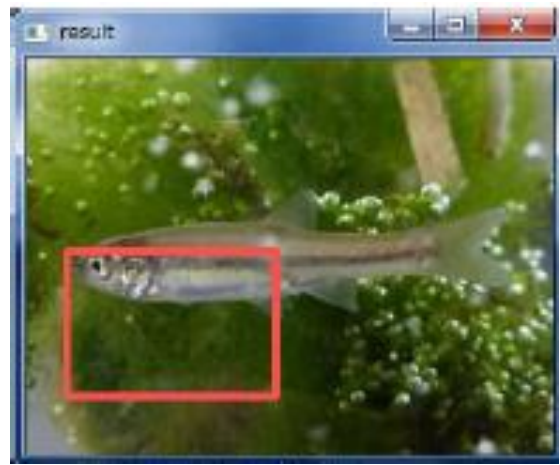

(4) Examples of false recognition

Fig.9: Result of the recognition of the entire body

Recognition rate and false recognition rate is shown in the following.

Recognition rate $=64 \%$

False recognition rate $=36 \%$

\subsection{Learning result of the face pattern}

In this experiment, 11 of 100 images are accurately recognized. Figure 10 show examples of the correct and the false recognition results.

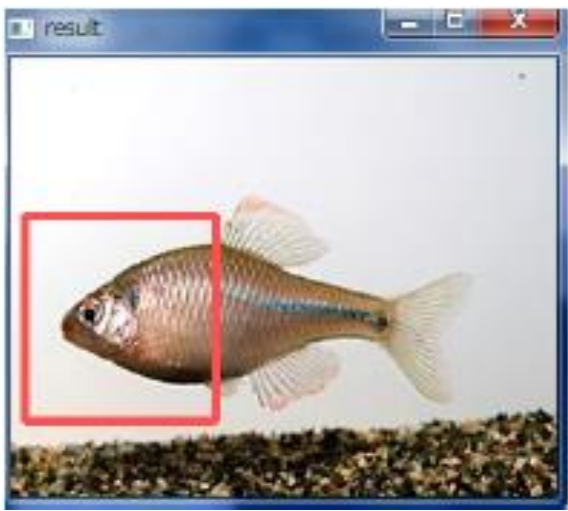

(1) Examples of recognition

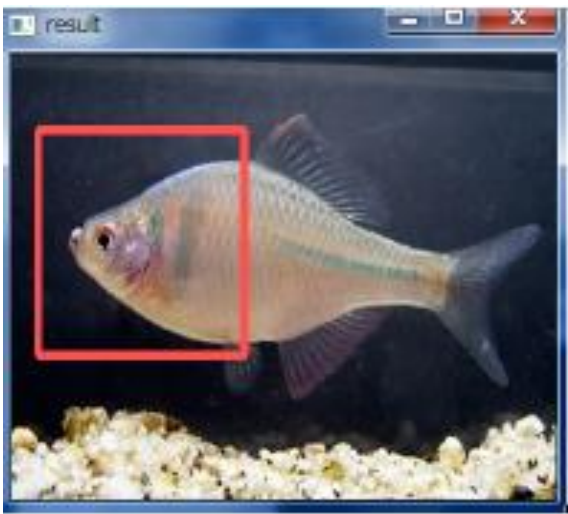

(2)Examples of recognition

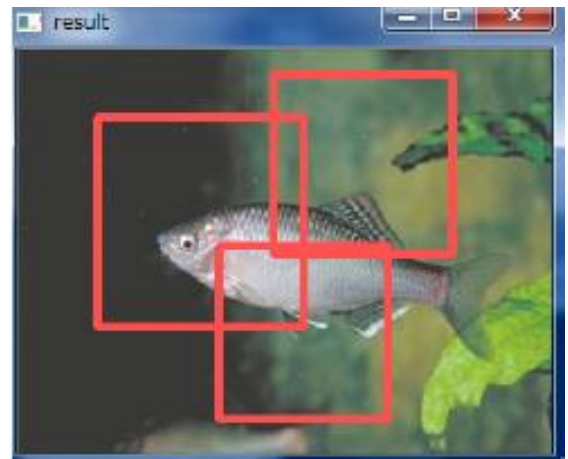

(3) Examples of false recognition 


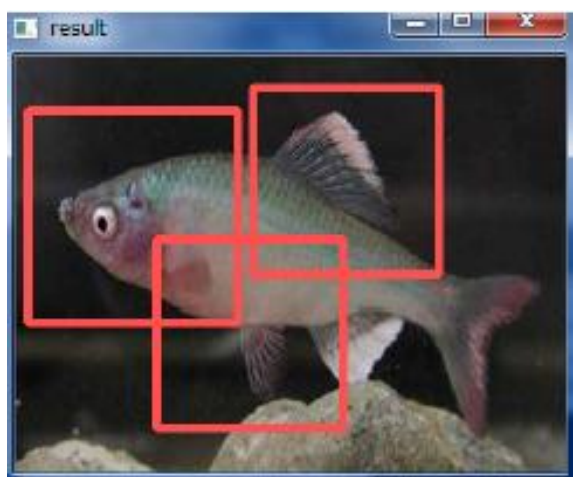

(5) Examples of false recognition

Fig.10: Result of the recognition of the face

Recognition rate and false recognition rate is shown in the following.

Recognition rate $=11 \%$

False recognition rate $=89 \%$

\subsection{Learning result of the fish's eye pattern}

In this experiment, only 1 images in 100 sheets is accurately recognized the eye. Figure 11 show examples of the correct and the false recognition results.

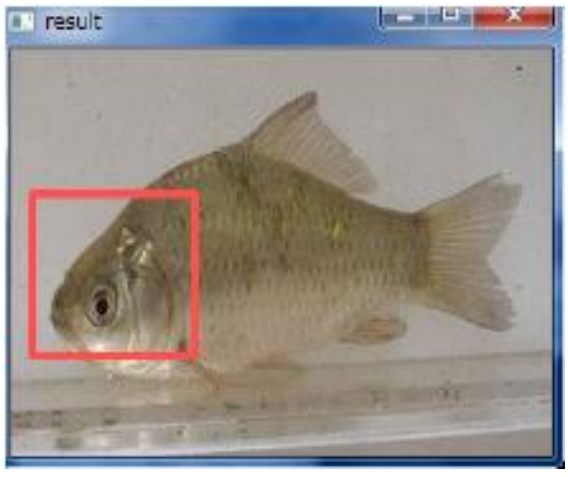

(1) Examples of recognition

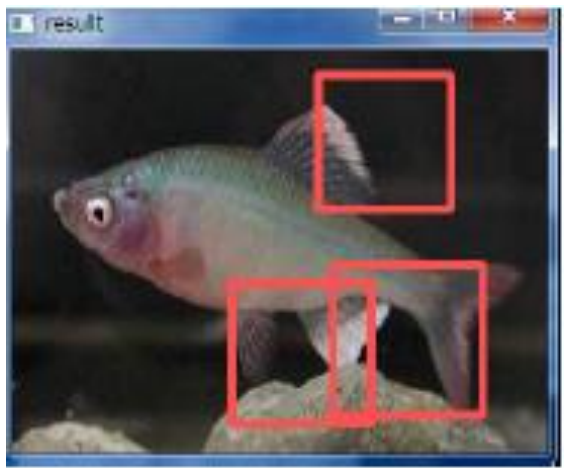

(2) Examples of false recognition

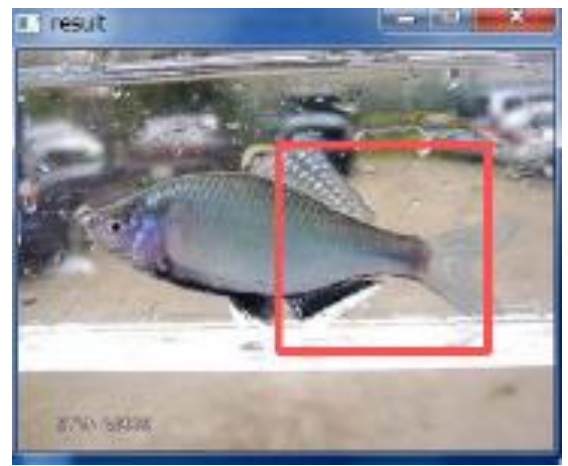

(3) Examples of false recognition

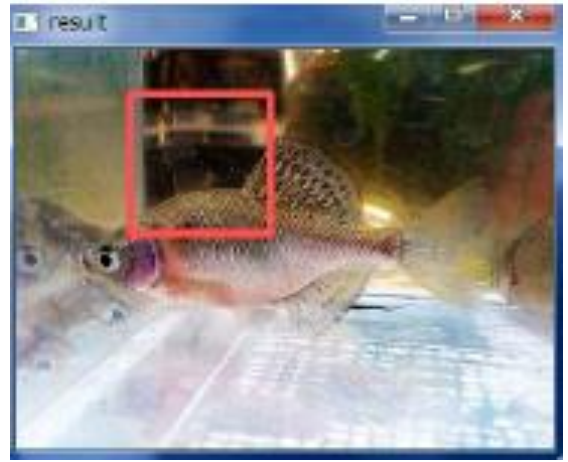

(4) Examples of false recognition

Fig.11: Result of the recognition of the eye

Recognition rate and false recognition rate is shown in the following.

Recognition rate $=1 \%$

False recognition rate $=99 \%$

\subsection{Learning result of the fish's body surface pattern}

In this experiment, 9 images in 100 sheets is accurately recognized the body surface. Figure 12 show examples of the correct and the false recognition results.

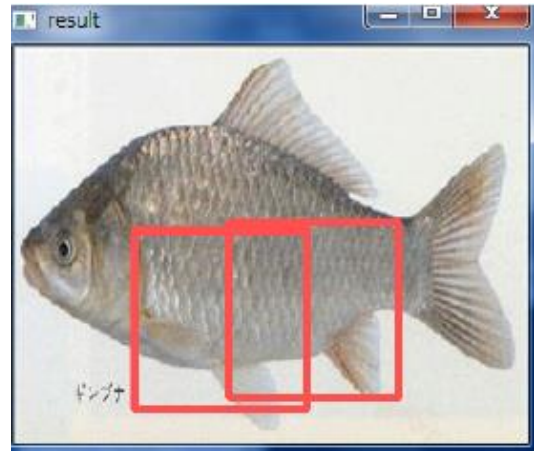


(1) Examples of recognition

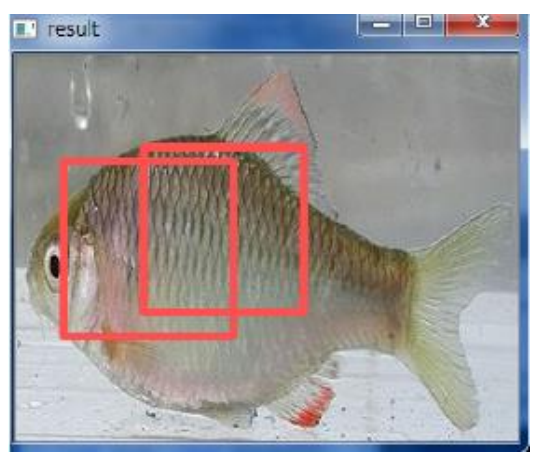

(2) Examples of recognition

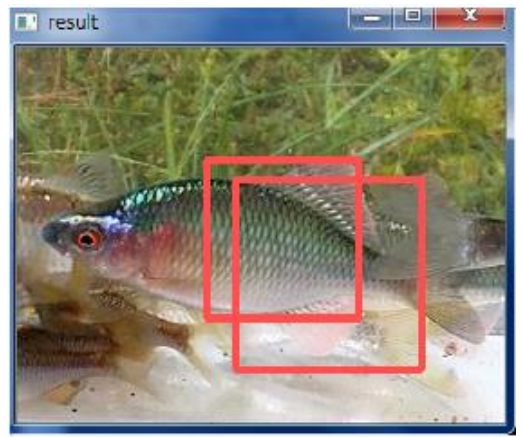

(3) Examples of recognition

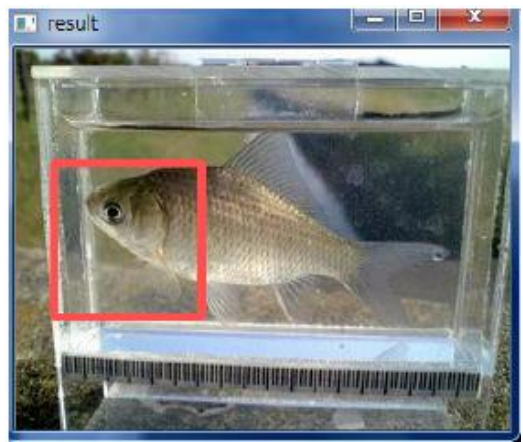

(4) Examples of false recognition

Fig.12: Result of the recognition of the body surface

Recognition rate and false recognition rate is shown in the following.

Recognition rate $=9 \%$

False recognition rate $=91 \%$

\subsection{Learning result of the fish's dorsal fin pattern}

In this experiment, only 2 images in 100 sheets is accurately recognized the dorsal fin. Figure 13 show examples of the correct and the false recognition results.

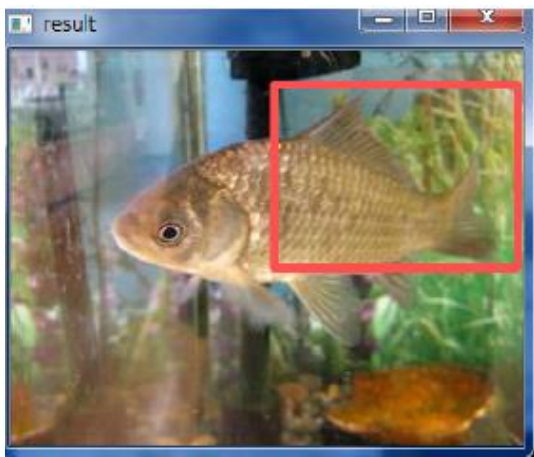

(1)Example of the result

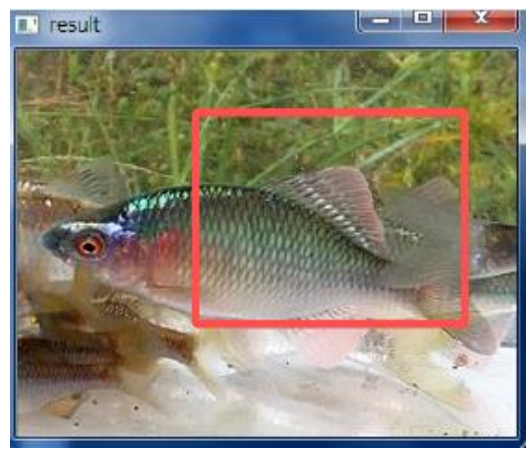

(2) Example of the result

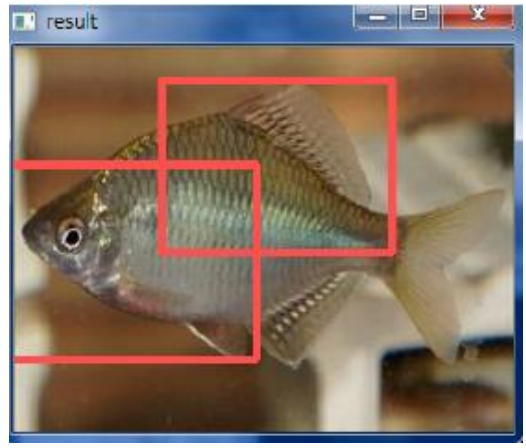

(3) Example of the result

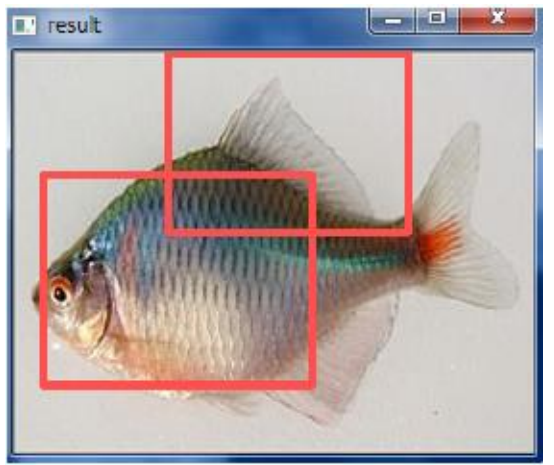

(4) Example of the result 
Recognition rate and false recognition rate is shown in the following.

Recognition rate $=2 \%$

False recognition rate $=98 \%$

It is shown in Table 1 above experimental results

Table 1. Experimental results.

\begin{tabular}{|c|c|c|}
\hline Site of fish & Recognition rate(\%) & False recognition rate(\%) \\
\hline Entire body & 64 & 36 \\
\hline Face & 11 & 89 \\
\hline Eye & 1 & 99 \\
\hline Body surface & 9 & 91 \\
\hline Dorsal fin & 2 & 98 \\
\hline
\end{tabular}

\section{Conclusions}

In this study, recognition rate using entire body learning data is the best. The accuracy is $64 \%$. Although this value is not high enough, it can detect the fish with a high probability if the fish pass through the camera more than once.

Furthermore, in future we are planning to make a hybrid judgment by combining the result with other body feature detectors to improve the reliability.

In this experiment, the pattern learning was performed with positive and negative images 1,000 pieces for each. This is not thought a enough number for machine learning, we should collect more samples in future work and make a more accuracy recognition result.

\section{References}

(1) Environmental issues of Lake Biwa: http://www.wwf.or.jp/activities/2009/09/679714.html,2 $015,7,30$

(2) Takeshi Mita : "The basic principles of AdaBoost and application to face detection: Tutorial Series(tutorial2) of CVIM study group ", IPSJ CVIM,[Vision of the computer and image media] , pp. 265-272, 2007

(3) Takahiro Ozaki, Yuji Yamauchi, Hironobu Fujiyoshi: "The vehicle detection by the two-stage AdaBoost using the Joint HOG feature", Moving image processing actual use of workshop (DIA2008), I1-13, 2008
(4) Hironobu Fujiyoshi : "The object detection by Joint features focused on the relevance of local features", IPSJ CVIM 166, pp.43-54.

(5) Human detection using HOG features and Boosting : http://www.vision.cs.chubu.ac.jp/joint_hog/pdf/HO G+Boosting LN.pdf, 2015, 6, 4 